\title{
Asymmetric simple exclusion process in one-dimensional chains with long-range links
}

\author{
Mina Kim ${ }^{1}$, Ludger Santen ${ }^{2}$ and Jae Dong Noh ${ }^{1,3}$ \\ 1 Department of Physics, University of Seoul, Seoul 130-743, Korea \\ 2 Theoretische Physik, Universität des Saarlandes, 66041 Saarbrücken, Germany \\ 3 School of Physics, Korea Institute for Advanced Study, Seoul 130-722, Korea \\ E-mail: jdnoh@uos.ac.kr
}

\begin{abstract}
We study the boundary-driven asymmetric simple exclusion process (ASEP) in a one-dimensional chain with long-range links. Shortcuts are added to a chain by connecting $p L$ different pairs of sites selected randomly where $L$ and $p$ denote the chain length and the shortcut density, respectively. Particles flow into a chain at one boundary at rate $\alpha$ and out of a chain at the other boundary at rate $\beta$, while they hop inside a chain via nearest-neighbor bonds and long-range shortcuts. Without shortcuts, the model reduces to the boundary-driven ASEP in a one-dimensional chain which displays the low density, high density, and maximal current phases. Shortcuts lead to a drastic change. Numerical simulation studies suggest that there emerge three phases; an empty phase with $\rho=0$, a jammed phase with $\rho=1$, and a shock phase with $0<\rho<1$ where $\rho$ is the mean particle density. The shock phase is characterized with a phase separation between an empty region and a jammed region with a localized shock between them. The mechanism for the shock formation and the nonequilibrium phase transition are explained by an analytic theory based on a meanfield approximation and an annealed approximation.
\end{abstract}

PACS numbers: 05.10.Ln, 05.60.-k, 64.60.-i, 68.35.Rh 


\section{Introduction}

The asymmetric simple exclusion process (ASEP) has been widely studied in the past decades 1. It is a nonequilibrium driven diffusive system of particles subject to the exclusion interaction. Despite its simplicity, the ASEP describes various nonequilibrium processes such as bio-polymerization [2], surface growth [3], traffic flow 4, 5, for example. Furthermore, the ASEP in one dimension is exactly solvable via the Bethe ansatz [6] and the matrix product ansatz [7] or direct solution of recursion relations [8]. The exact solution contributes to deeper understanding of fluctuation phenomena [9, 10] and nonequilibrium phase transitions [8].

Most studies on the ASEP have been performed on one-dimensional (1D) chains. On the other hand, there are many quasi-1D systems which involve long-range links. If a polymer chain folds randomly, there arise contacts between different polymer segments which are far apart along a backbone [11]. Those contacts can be regarded as long-range links for a transport process on a network of polymer chains. A gene regulatory protein diffuses along the DNA chain to search for its target gene [12. It can make a long-range jump by dissociation from and reassociation with the DNA. Recent studies show that real world traffic networks have a complex structure with long-range links [13, 14]. In this respect, interests are growing in the study of the ASEP on complex networks [15, 16, 17.

In this work, we address the question: What is the transport capacity of a complex network for particles interacting via mutual exclusion? In order to contribute to this issue we consider the ASEP on a 1D chain with long-range links, called shortcuts, for open boundary conditions. It will turn out that the shortcuts cause a drastic change in the phase diagram of the ASEP.

We start with a brief review of the 1D ASEP. The original model is defined on a 1D lattice. The lattice sites are either occupied by at most one particle or empty. Multiple occupancy on a site is prohibited [exclusion interaction]. Particles may hop to the left and right with a bias to one direction [ASEP]. A closely related equilibrium process is the so-called symmetric simple exclusion process (SSEP) where particles hop in both directions with equal rates. For periodic boundary conditions, the model has a trivial steady state where every microscopic configuration is equally likely irrespective of the hopping bias [6]. However, the bias is relevant for the dynamic scaling behavior. In the context of growing interfaces, the SSEP belongs to the Edward-Wilkinson (EW) universality class [18, and the ASEP to the Kardar-Parisi-Zhang (KPZ) universality class [19. Both classes are characterized by the power-law scaling $\tau \sim \xi^{z}$ between characteristic time and length scales. The scaling exponent is given by $z=2$ for the EW class and $z=3 / 2$ for the KPZ class [6, 20].

For open boundary conditions the chain is coupled to particle reservoirs at both ends. One acts as a particle source emitting particles at a rate $\alpha$, and the other as a sink absorbing particles at a rate $\beta$. Interestingly, the system driven by the open boundaries displays nonequilibrium phase transitions between low-density (LD), highdensity (HD), and maximal-current (MC) phases [7, 8. The system belongs to the LD phase if the capacity of the particle source $\alpha$ controls the particle flux, i.e. if $\alpha<\beta$ and $\alpha<p_{h} / 2$, where $p_{h}$ denotes the hopping rate of the particles. The system belongs to the HD phase when the outgoing rate $\beta$ is smaller than $\alpha$ and $p_{h} / 2$. The overall particle density is determined by the outgoing rate $\beta$ and there is a macroscopic congestion of particles. When both $\alpha$ and $\beta$ are larger than $p_{h} / 2$, the capacity of the system is limited by the capacity of the bulk. Such a phase is called the MC phase, 
where the overall particle density is independent of $\alpha$ and $\beta$.

In the 1D ASEP, particle hopping is a short-ranged process between neighboring sites. There are a few attempts to study the effect of a long-range hopping on the phase diagram of the ASEP for open boundary conditions. Szavits-Nossan and Uzelac considered the ASEP with a probabilistic long-range hopping [15]. A particle can hop to any site at a distance $l$ with probability $p_{l} \sim 1 / l^{\sigma+1}$. They obtained that the phase diagram remains the same as that of the 1D ASEP for $\sigma>1$. For $\sigma \leq 1$, however, the system does not display any phase transition. Ha et al. considered a boundary-driven 1D ASEP model where particles may perform a short-range hopping between nearestneighbor sites or a long-range hopping [16. Upon a long-range hopping, a particle jumps to an empty site directly behind a next particle in front of it. It was found that the long-range hopping introduces an instability towards a so-called empty-road phase. Otwinowski and Boettcher considered the ASEP on a one-dimensional chain decorated with hierarchically organized long-range links [17. This model displays the LD and HD phases. Besides, depending on the way the long-range hopping is implemented, an intermediate phase may also be realized.

In this work, we investigate the role of long-range hoppings in a driven system in a generic setting. For that purpose, we study the ASEP on a 1D chain with open boundary conditions and additional long range links connecting randomly-selected pairs of sites. The result is a graph similar to a small-world network [13. Additionally, we couple this network to two particle reservoirs, a particle source connected to one boundary site and sink to another. In contrast to the 1D ASEP, particle source and sink now are connected via the small-world network. This setup describes a generic scenario for directed transport through a complex network with limited capacity. It is generally believed that long-range links make a system defined on a small-world network homogeneous 21. In contrast, our study shows that the system develops a localized shock which separates a 1D backbone into an empty region near the entrance and a fully-occupied region near the exit. Such an inhomogeneity is caused by the interplay between the boundary driving and the long-range hopping. The steady-state position of the shock depends on the particle input and output rates, which results in an interesting phase diagram.

This paper is organized as follows: In Sec. 2, we introduce a boundary-driven ASEP in a 1D chain with long-range links. In Sec. 3, we present numerical results obtained from Monte Carlo simulations. Measuring the overall particle density $\rho$, we obtain a numerical phase diagram which consists of an empty $(\mathrm{E})$ phase, a jammed $(\mathrm{J})$ phase, and a shock (S) phase. The overall particle density takes the value of $\rho=0(1)$ in the $\mathrm{E}(\mathrm{J})$ phase, while it varies continuously in the $\mathrm{S}$ phase. The shock phase is characterized by a localized shock which separates a 1D backbone into empty and jammed domains. In order to understand the mechanism for the shock formation and the phase transition, we develop an approximate analytic theory. This is presented in Sec. 4. We summarize and conclude the paper in Sec. 5.

\section{Boundary-driven ASEP with long-range links}

Consider a graph $\mathcal{G}$ consisting of $L$ sites which are labeled as $i=1,2, \cdots, L$. Every pair $(i, i+1)$ with $i=1, \cdots, L-1$ is connected with a short-range link to form a $1 \mathrm{D}$ backbone. In addition, we select $p L$ pairs of sites at random and add long-range links between them (see Figure 1). The shortcut density, the total number of long-range links divided by $L$, is denoted by $p$. Every link has an orientation: A link between $i$ 


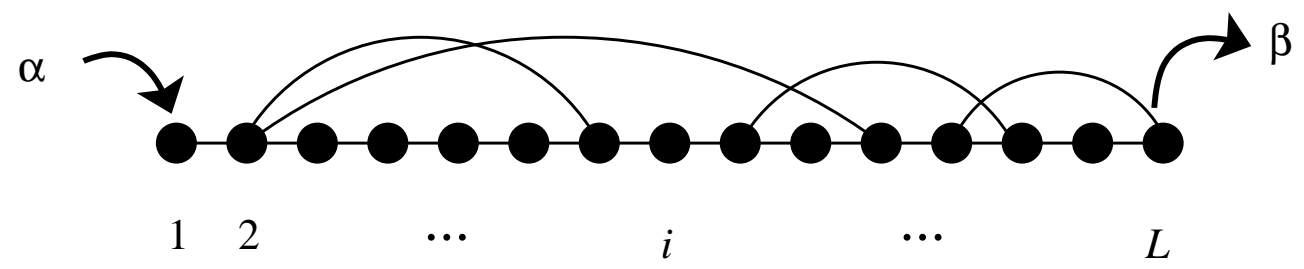

Figure 1. A graph consisting of $L$ sites, short-range links, and long-range links. The site $i=1$ and $L$ are attached to a particle source and sink, respectively.

and $j$ is directed from $i$ to $j$ if $i<j$ and vice versa. Such a direction will be referred to as a forward direction. It is assumed that the boundary site $i=1$, called an entrance, is attached to a particle source where particles are fed into the system at constant rate. The other boundary site $i=L$, called an exit, is attached to a particle sink which absorbs particles at a constant rate.

We consider the ASEP process on such a graph. More precisely the particle dynamics is defined as follows: A particle enters the system at a rate denoted as $\alpha$. Particles which have entered the system are then allowed to hop along forward links at rate one. If a particle is located at a site which is connected to others via multiple forward links one of these is randomly chosen. For each forward link the selection probability is given by $1 / k_{i}$ where $k_{i}$ denotes the number of forward links at a given site $i$. A particle can leave the system at site $L$ (the exit) at a rate denoted as $\beta$. Throughout the process, particles are subject to the exclusion principle which forbids multiple occupancy. Therefore any trial move violating the exclusion principle is rejected.

One can represent the structure of a graph $\mathcal{G}$ with an adjacency matrix $\boldsymbol{A}$ whose elements $A_{i j}$ take the value of 1 only if there is a forward link from site $j$ to $i$. Due to the link directionality, $A_{i j}=0$ for all $i<j$. Then, the hopping probability of a particle from site $i$ to $j$ is given by

$$
u_{j i}=\frac{A_{j i}}{k_{i}}
$$

where $k_{i} \equiv \sum_{l>i} A_{l i}$ denotes the number of forward links out of site $i$. It should be noticed that the hopping probability is a quenched random variable. It varies from one realization of a graph to another. Consequently, the quenched average over different realizations of the graph is necessary.

Here, we are interested in the particle density distribution and the current. Let $n_{i}(=0,1)$ be the occupation number at site $i$. It is a stochastic variable evolving according to the ASEP dynamics. For a given realization of the graph, its average value is governed by the time evolution equation

$$
\frac{d}{d t}\left\langle n_{i}\right\rangle=\sum_{j<i} u_{i j}\left\langle n_{j}\left(1-n_{i}\right)\right\rangle-\sum_{j>i} u_{j i}\left\langle n_{i}\left(1-n_{j}\right)\right\rangle
$$

for $1<i<L$ and

$$
\begin{aligned}
\frac{d}{d t}\left\langle n_{1}\right\rangle & =\alpha\left(1-\left\langle n_{1}\right\rangle\right)-\sum_{j=2}^{L} u_{j 1}\left\langle n_{1}\left(1-n_{j}\right)\right\rangle \\
\frac{d}{d t}\left\langle n_{L}\right\rangle & =\sum_{j=1}^{L-1} u_{L j}\left\langle n_{j}\left(1-n_{L}\right)\right\rangle-\beta\left\langle n_{L}\right\rangle .
\end{aligned}
$$


Here the angle bracket $\langle(\cdot)\rangle$ represents the average of a quantity $(\cdot)$ for different realizations of the stochastic noise.

In contrast to the 1D-ASEP particle conservation in the bulk does not imply that the current is the same for every link. Therefore, the current can be defined in several ways. An obvious choice is to count the number of particles entering or leaving the system since the particle reservoirs are each connected via a single link. The incoming current $J_{\text {in }}$ through the entrance and the outgoing current $J_{\text {out }}$ from the exit are given by

$$
\begin{aligned}
& J_{\text {in }}=\alpha\left(1-\left\langle n_{1}\right\rangle\right), \\
& J_{\text {out }}=\beta\left\langle n_{L}\right\rangle .
\end{aligned}
$$

In the bulk site $i(=1, \cdots, L-1)$, the current $J_{i}$ is defined as the total current of particles departing from sites $j=1, \cdots, i$ and arriving at sites $l=i+1, \cdots, L$. It is given by

$$
J_{i}=\sum_{j=1}^{i} \sum_{l=i+1}^{L} u_{l j}\left\langle n_{j}\left(1-n_{l}\right)\right\rangle .
$$

With this definition of the current, the time evolution of the density is given by $d\left\langle n_{i}\right\rangle / d t=J_{i-1}-J_{i}$ for $1<i<L$ and $d\left\langle n_{1}\right\rangle / d t=J_{\text {in }}-J_{1}$ and $d\left\langle n_{L}\right\rangle / d t=J_{L-1}-J_{\text {out }}$. Hence, all values of the local currents $J_{i}, J_{\text {in }}$ and $J_{\text {out }}$ should be the same in the steady state, where $d\left\langle n_{i}\right\rangle / d t=0$.

The time-evolution equations (21), (3), and (4) already illustrate the difficulty in calculating the quantities of interest. In order to determine the particle density twopoint correlations have to be calculated, which in turn require higher order correlation functions. Furthermore, the quenched average over the random variables $\left\{u_{i j}\right\}$ is necessary. An exact solution of this process is not available. So, we will investigate the model using a numerical simulation method in the following section.

\section{Simulation Results}

In this section we present numerical results obtained from Monte Carlo simulations. For a simulation, one generates a graph $\mathcal{G}$ with an adjacency matrix $\mathbf{A}$ consisting of a $1 \mathrm{D}$ chain of $L$ sites and $p L$ shortcuts. Particles on $\mathcal{G}$ move in the following way: First, we select a random variable $l \in\{0,1, \cdots, L\}$ with equal probability. (i) If $l=0$, we add a particle to the entrance site $i=1$ with probability $\alpha$ if it is empty. (ii) If $0<l<L$, we try to move a particle (if any) at site $l$ to a target site $j$ selected among $\{l+1, \cdots, L\}$ with probability $u_{j l}$ given in (1). The particle move can be carried out if the target site is empty. (iii) If $l=L$, we remove a particle (if any) at the exit site $i=L$ with probability $\beta$. The time is incremented by unity after $(L+1)$ trials.

We are interested in the particle distribution and the current in the steady state. After a transient period of time interval $T_{t}$, we average the occupation number for the time interval $T_{s}$ to obtain the steady-state occupation number distribution $\left\langle n_{i}\right\rangle$. Note that the mean occupation number varies from one realization of a graph to another. In a second step a quenched average $[(\cdots)]_{\mathcal{G}}$ over graph realizations is necessary. The quenched averaged quantities will be denoted as

$$
\rho_{i}=\left[\left\langle n_{i}\right\rangle\right]_{\mathcal{G}} .
$$



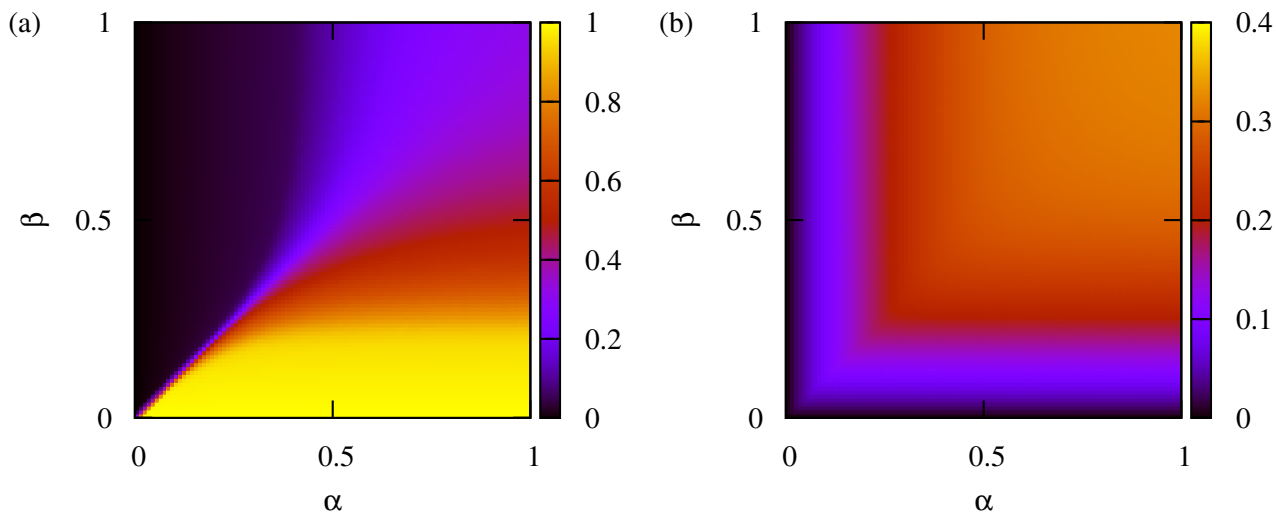

Figure 2. (Color online) Particle density $\rho$ (a) and current $J$ (b) in the $\alpha \beta$ plane. The system size is $L=400$ and the shortcut density $p=0.2$.

The overall particle density is given by

$$
\rho=\frac{1}{L} \sum_{i=1}^{L} \rho_{i}
$$

The steady-state current is given by

$$
J=\alpha\left(1-\rho_{1}\right)=\beta \rho_{L} .
$$

The overall behavior of the particle density $\rho$ and the current $J$ is presented in Figure 2 Those data were averaged over $N_{s}=1000$ graph realizations over a time interval $T_{s}=500000$ after a transient interval $T_{t}=10000$. Figure 2 suggests that there exist three different regimes. When $\alpha$ is small, the overall density $\rho$ is close to zero. On the other hand, $\rho$ is close to 1 when $\beta$ is small. A finite-size-scaling (FSS) analysis reveals that the system indeed undergoes nonequilibrium phase transitions between three different phases, which will be discussed below.

Figure 3(a) shows the plot of $(1-\rho)$ along a line $\alpha=0.8$ at several values of $L=100, \cdots, 3200$. Those data are well fitted to a FSS form 22

$$
(1-\rho)=L^{-x_{v}} \mathcal{F}_{v}\left(\left(\beta-\beta_{c}\right) L^{1 / \nu_{v}}\right)
$$

with $\beta_{c} \simeq 0.15, x_{v} \simeq 0.4$, and $\nu_{v} \simeq 2.5$. The scaling function has a limiting behavior $\mathcal{F}_{v}(y \gg 1) \sim y^{\beta_{v}^{\prime}}$ with $\beta_{v}^{\prime}=x_{v} \nu_{v} \simeq 1.0$. The FSS indicates that system undergoes a continuous phase transition at a critical point $\beta=\beta_{c}$. For $\beta<\beta_{c}$, the density of vacant sites is zero. That is to say, the system is fully occupied by particles. Such a macroscopic state will be called a jammed phase. Near the critical point with $\epsilon=\beta-\beta_{c}$, the density of empty sites scales as

$$
(1-\rho) \sim \epsilon^{\beta_{v}^{\prime}} .
$$

Figure 3(b) shows the plot of $\rho$ along a line $\beta=0.8$. The data are well fitted to a FSS form [22]

$$
\rho=L^{-x_{p}} \mathcal{F}_{p}\left(\left(\alpha-\alpha_{c}\right) L^{1 / \nu_{p}}\right)
$$

with $\alpha_{c} \simeq 0.36, x_{p} \simeq 0.5$, and $\nu_{p} \simeq 2$. The scaling function has a limiting behavior $\mathcal{F}_{p}(y \gg 1) \sim y^{\beta_{p}^{\prime}}$ with $\beta_{p}^{\prime}=x_{p} \nu_{p} \simeq 1.0$. Hence we conclude that the system undergoes 

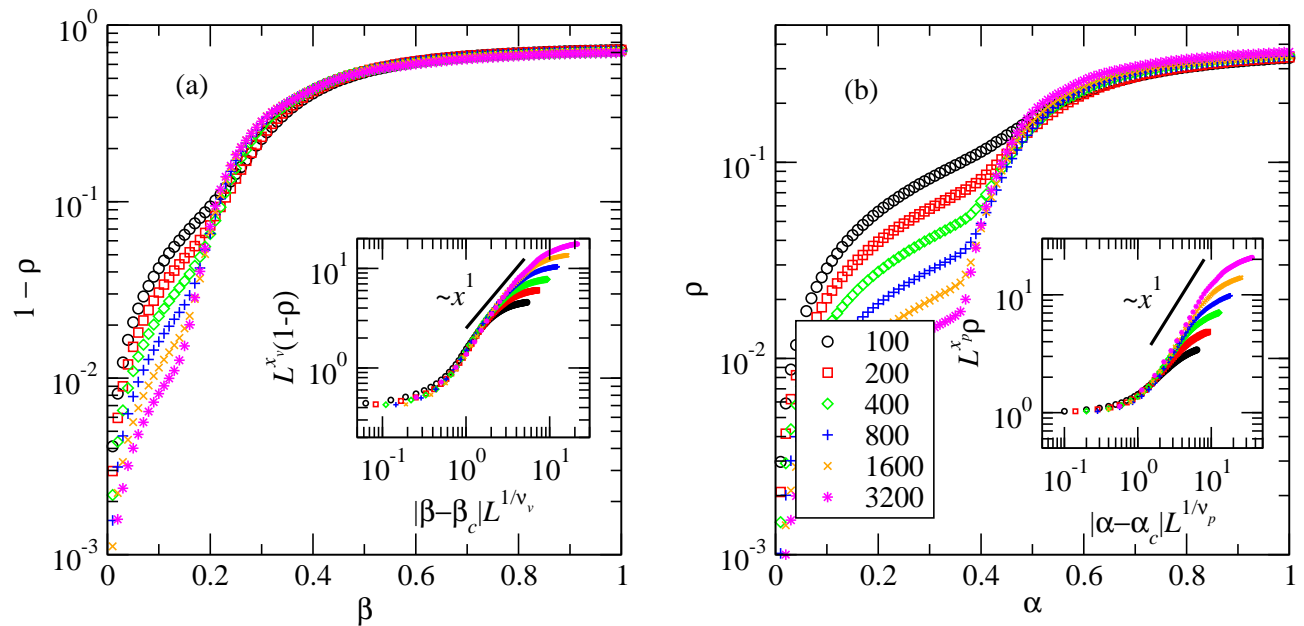

Figure 3. (Color online) (a) Plot of the density of empty sites vs. $\beta$ at fixed $\alpha=0.8$. (b) Plot of the particle density vs. $\alpha$ at fixed $\beta=0.8$.

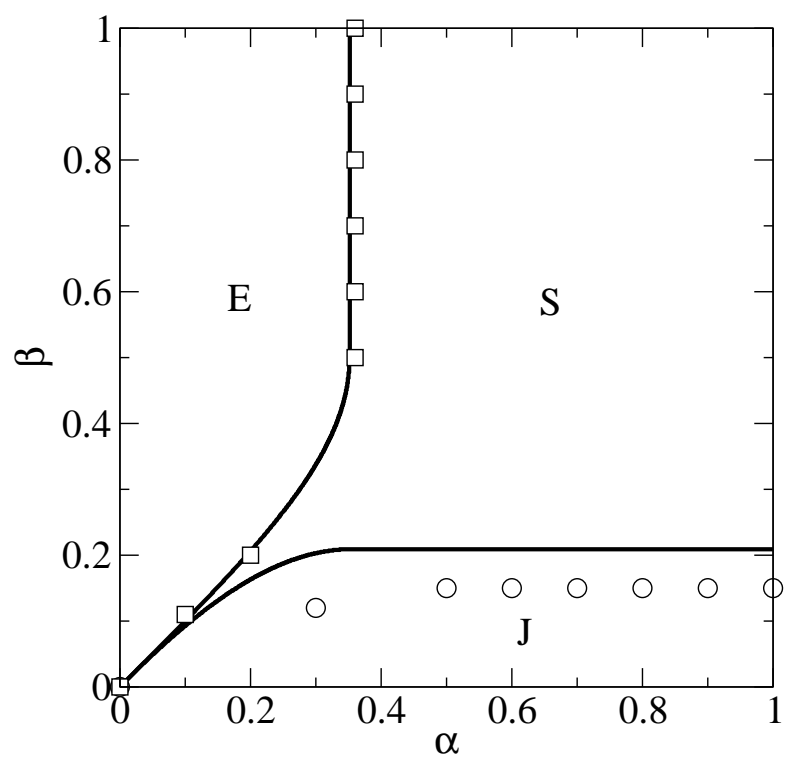

Figure 4. Phase diagram at $p=0.2$. The location of symbols ( $\square$ between the $\mathrm{E}$ and $\mathrm{S}$ phases, and $\circ$ between the $\mathrm{S}$ and J phases) is determined from the FSS analysis. The solid lines are the phase boundary obtained from the mean-field and annealed approximation developed in Sec. 4

a continuous phase transition at a critical point $\alpha=\alpha_{c}$. The particle density vanishes for $\alpha<\alpha_{c}$. Such a macroscopic state will be called an empty phase. Near the critical point with $\epsilon=\alpha-\alpha_{c}$, the particle density scales as

$$
\rho \sim \epsilon^{\beta_{p}^{\prime}} .
$$

Repeating the FSS analysis, we obtain the numerical phase diagram as shown in Figure 4 The phase diagram consists of the empty (E) phase with $\rho=0$ and the 

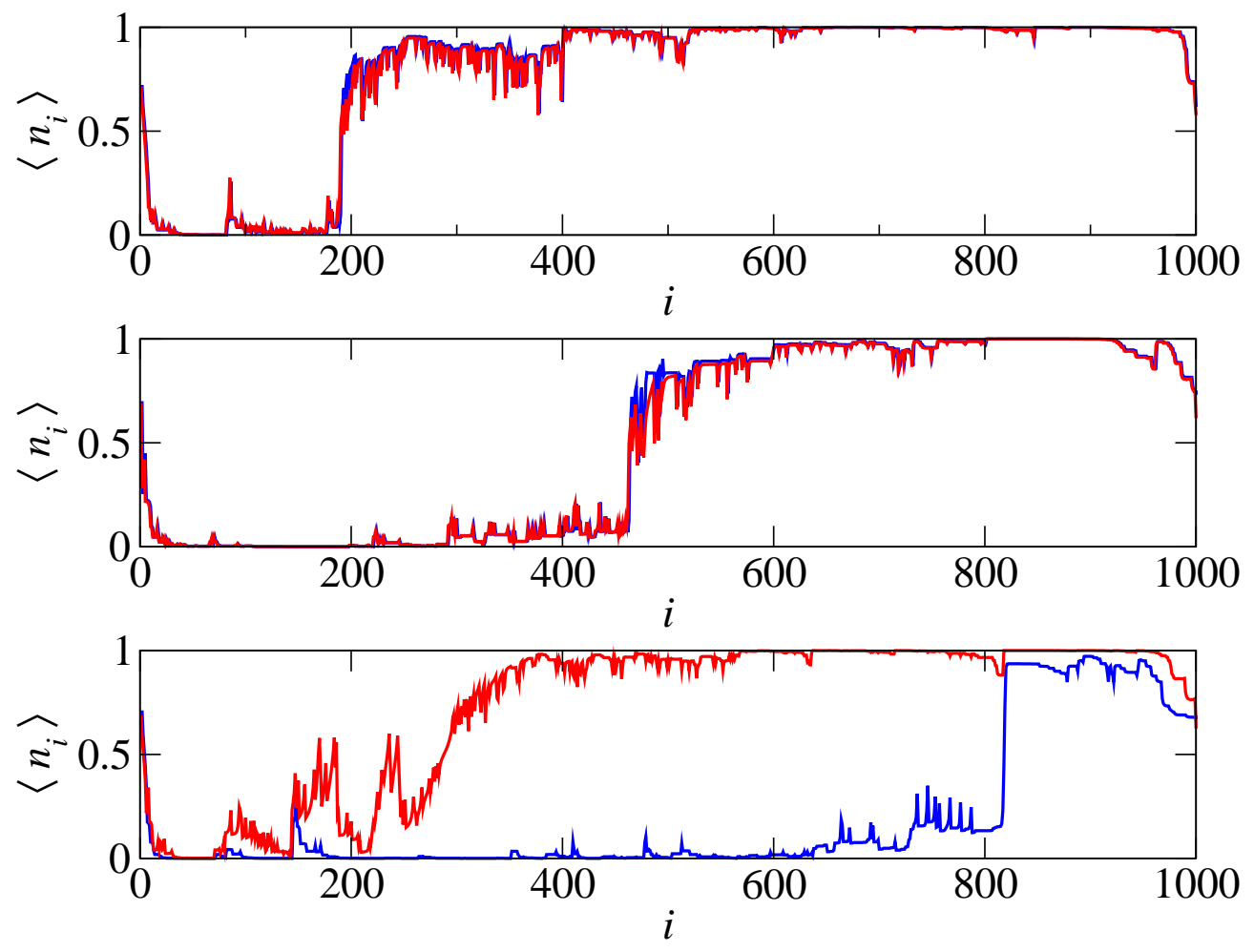

Figure 5. (Color online) Particle density profile in the shock phase. Each panel contains a data set obtained from a different realization of a graph. Monte Carlo simulation data are drawn with a red line. Also shown with a blue line are numerical data from the mean field approximation as explained in Sec. 4 Parameter values are $L=1000, p=0.2, \alpha=1.0$, and $\beta=0.5$.

jammed $(\mathrm{J})$ phase with $\rho=1$. The other phase with $0<\rho<1$ will be called a shock $(\mathrm{S})$ phase. Although the phase diagram looks similar to that of the ASEP on 1D chains [7, 8, the nature of the phases is different.

The particle distribution is intriguing in the $\mathrm{S}$ phase. Taking $\alpha=1.0$ and $\beta=0.5$, we have measured the particle density distribution $\left\{\left\langle n_{i}\right\rangle\right\}$ in the steady state at a given realization of $\mathcal{G}$. Figure 5 shows typical distributions. There is a phase separation between a region with $\left\langle n_{i}\right\rangle \simeq 0$ and a region with $\left\langle n_{i}\right\rangle \simeq 1$.0. The domain boundary between the two regions is called a shock. The localized shock gives a hint why there are phase transitions into the $\mathrm{E}$ and $\mathrm{J}$ phases. The system can be in the $\mathrm{E}(\mathrm{J})$ phase when the shock is absorbed at the exit (entrance). Hence it is crucial to understand the mechanism for the shock formation. This will be discussed in the following section.

Before proceeding, we present a physical argument for the phase diagram. It is known that the diameter $l_{D}$ of the small-world network with nonzero shortcut density $p$ scales as $l_{D} \sim \frac{1}{p} \ln (p L)$ [14. This means that any site can be reached from a given site within $l_{D}$ steps [13, 14. Then, a particle injected at the entrance can move toward the exit with a diverging speed $v \simeq L / l_{D} \sim L / \ln L$ along the backbone in the $L \rightarrow \infty$ limit when $\alpha$ is so small that the exclusion is irrelevant near the entrance. Consequently, particles can escape instantly from the system and the E phase can be 
realized. Using the same argument we can understand the origin of the $\mathrm{J}$ phase. A hopping of a particle from one site to another is equivalent to a hopping of a hole in the opposite direction. Hence, when $\beta$ is small enough, a hole injected at the exit can travel toward the entrance with the diverging speed in $\mathcal{O}(\ln L)$ steps and the $\mathrm{J}$ phase can be realized. The $\mathrm{S}$ phase is a result of competition between the empty domain (which is stabilized by the long-range hopping of particles) and the jammed domain (which is stabilized by the long-range hopping of holes). This argument clearly shows that the small-world property is crucial in the formation of the E, J, and $\mathrm{S}$ phases. The small-world property emerges at any nonzero value of $p$ [14. Hence we expect that those phases replace the $\mathrm{LD}, \mathrm{HD}$, and $\mathrm{MC}$ phases of the conventional ASEP in 1D chains immediately as one turns on the long-range links.

We have also performed the numerical simulations with $p=0.1$ and $p=1.0$. In both cases we observe qualitatively the same phase diagram. As $p$ increases, the $\mathrm{E}$ phase expands while the $\mathrm{J}$ phase shrinks. For example, when $\beta=0.8$, we found that $\alpha_{c}=0.35,0.36$, and 0.39 at $p=0.1,0.2$, and 1.0, respectively. When $\alpha=0.8$, we found that $\beta_{c}=0.20,0.15$, and 0.07 at $p=0.1,0.2$, and 1.0 , respectively. We do not find a simple explanation for the observed $p$ dependence of the phase boundary.

\section{Analytic Results}

\subsection{Mean field approximation}

We adopt a mean field approximation by assuming that

$$
\left\langle n_{i} n_{j}\right\rangle=\left\langle n_{i}\right\rangle\left\langle n_{j}\right\rangle
$$

for all $i$ and $j$. Applying the mean field approximation to (2), (3), and (4) yields that

$$
\frac{d}{d t}\left\langle n_{i}\right\rangle=-\left\langle n_{i}\right\rangle R_{i}+\left(1-\left\langle n_{i}\right\rangle\right) Q_{i},
$$

where the auxiliary quantities are defined as

$$
R_{i}=\beta \delta_{i, L}+\left(\sum_{j>i} u_{j i}\left(1-\left\langle n_{j}\right\rangle\right)\right)\left(1-\delta_{i, L}\right)
$$

and

$$
Q_{i} \equiv \alpha \delta_{i, 1}+\left(\sum_{j<i} u_{i j}\left\langle n_{j}\right\rangle\right)\left(1-\delta_{i, 1}\right)
$$

with the Kronecker $\delta$ symbol. The quantities $R_{i}$ and $Q_{i}$ can be interpreted as particle evaporation and deposition rates at site $i$, respectively. They depend on the structure of an underlying graph through $\left\{u_{i j}\right\}$ and the whole particle density distribution $\left\{\left\langle n_{i}\right\rangle\right\}$. This interpretation of the rates $R_{i}$ and $Q_{i}$ allows to relate our model to the 1D ASEP with constant evaporation and deposition rates which was studied in 23, 24, 25. In contrast to that model the rates $R_{i}$ and $Q_{i}$ in our model depend on the particle distribution as well as on the realization of the adjacency matrix.

The steady-state density satisfying $d\left\langle n_{i}\right\rangle / d t=0$ is given by

$$
\left\langle n_{i}\right\rangle=\frac{Q_{i}}{R_{i}+Q_{i}} .
$$

Since $R_{i}$ and $Q_{i}$ depends on the particle distribution, (19) should be solved selfconsistently. The self-consistent equation can be solved numerically via iteration. 
Starting from any trial distribution, one updates it by evaluating the right-hand side of (19). A particle distribution converges to a steady-state distribution without difficulty.

In order to test the mean field approximation, we compare the particle density profile obtained from the Monte Carlo method and the mean field approximation. For a given graph $\mathcal{G}$, we have performed the Monte Carlo simulation and solved the self-consistent equation for the steady-state density profile. They are compared in Figure 5 . The mean field approximation reproduces the phase separation and the shock in the density profile. In many cases, a mean field result is remarkably close to a Monte Carlo result (see the top and middle panels in Figure 5). On the other hand, in some cases, there is a noticeable quantitative discrepancy between them (see the bottom panel in Figure 5). Nevertheless, the mean field result still indicates the presence of the shock clearly. Therefore, we conclude that the mean field theory is suitable for the description of the phase transitions.

\subsection{Annealed network approximation}

The density profile given by the solution of (19) depends on a graph realization $\mathcal{G}$. We have to perform the quenched average over graph realizations to obtain the disorder-averaged density profile $\rho_{i}=\left[\left\langle n_{i}\right\rangle\right]_{\mathcal{G}}$. The quenched average is analytically intractable. Hence we further make an approximation by replacing an adjacency matrix element $A_{i j}$, which is a quenched random variable, with its disorderaveraged value $\left[A_{i j}\right]_{\mathcal{G}}$. Such an approximation is called an annealed approximation. The annealed approximation is useful in studying physical systems on graphs or networks [26, 27, 28,.

On a graph of $L$ sites, there are $p L$ long-range links. Hence the probability to find a long-range links between two sites $i$ and $j \neq i \pm 1$ is given by $p_{1}=$ $2 p L /((L-1)(L-2)) \simeq 2 p / L$. Short-range links are connecting sites $i$ and $i+1$ for $i=1, \cdots, L-1$. Taking account of the short- and long-range links, we obtain that

$$
\left[A_{i j}\right]_{\mathcal{G}}=\delta_{i, j+1}+\left(1-\delta_{i, j+1}\right) \frac{2 p}{L}
$$

for $i>j$ and $\left[A_{i j}\right]_{\mathcal{G}}=0$ for $i \leq j$. The parameter $u_{i j}$ is replaced by $u_{i j}=\left[A_{i j}\right]_{\mathcal{G}} /\left[k_{j}\right]_{\mathcal{G}}$ with $\left[k_{j}\right]_{\mathcal{G}}=(1+2 p(L-j-1) / L)$.

In the annealed approximation, the self-consistent equation for the disorderaveraged density $\rho_{i}$ becomes

$$
\rho_{i}=\frac{Q_{i}}{R_{i}+Q_{i}}
$$

where $R_{i}$ and $Q_{i}$ are given by

$$
\begin{aligned}
& R_{i}=1-\frac{1}{1+\frac{2 p}{L}(L-i-1)}\left(\rho_{i+1}+\frac{2 p}{L} \sum_{j=i+2}^{L} \rho_{j}\right) \\
& Q_{i}=\frac{\rho_{i-1}}{1+\frac{2 p}{L}(L-i)}+\sum_{j=1}^{i-2} \frac{\frac{2 p}{L} \rho_{j}}{1+\frac{2 p}{L}(L-j-1)}
\end{aligned}
$$

with the boundary terms $R_{L}=\beta$ and $Q_{1}=\alpha$. Equations (21), (22), and (23) are the starting point for further analysis. 


\subsection{Shock state}

We first consider the sites with $i=\mathcal{O}(1)$ near the entrance. Ignoring $\mathcal{O}\left(L^{-1}\right)$ corrections, one can approximate $R_{i}$ and $Q_{i}$ as

$$
\begin{aligned}
R_{i}^{i n} & =\frac{1}{1+2 p}\left(1-\rho_{i+1}\right)+\frac{2 p}{1+2 p}(1-\rho) \\
Q_{i}^{i n} & =\frac{1}{1+2 p} \rho_{i-1}
\end{aligned}
$$

with the boundary term $Q_{1}^{i n}=\alpha$ and the overall particle density $\rho$. These expressions allow for an interpretation for an effective dynamics near the entrance: A particle at site $i$ performs a short-range jump to site $i+1$ with probability

$$
W_{h}^{i n}=\frac{1}{1+2 p}
$$

or annihilates spontaneously with the probability

$$
W_{a}^{i n}(\rho)=\frac{2 p}{1+2 p}(1-\rho) .
$$

Due to the effective annihilation, the particle density should decay to zero exponentially with the distance from the entrance along the backbone with a characteristic length scale $\xi_{\text {in }}=1 / W_{a}^{i n}(\rho)$ unless $\rho=1$. This feature is consistent with the density profile shown in Figure 5 .

We next consider the sites $i=L-l$ with $l=\mathcal{O}(1)$ near the exit. Ignoring again $\mathcal{O}\left(L^{-1}\right)$ corrections, one can approximate $R_{i}$ and $Q_{i}$ by

$$
\begin{aligned}
R_{i}^{\text {out }} & =1-\rho_{i+1} \\
Q_{i}^{\text {out }} & =\rho_{i-1}+\frac{2 p}{L} \sum_{j=1}^{i-2} \frac{\rho_{j}}{1+2 p(L-j-1) / L}
\end{aligned}
$$

with the boundary term $R_{L}^{\text {out }}=\beta$. These expressions suggest that particles near the exit have a following effective dynamics: A particle at site $i$ performs a short-range hopping to site $i+1$ with the probability

$$
W_{h}^{\text {out }}=1 \text {, }
$$

and particles are created spontaneously at each site with the probability given by the second term in (29). Due to the creation, the particle density should saturate to unity as one departs from the exit along the backbone.

The density profiles stemming from the both boundaries converge to different values of 0 and 1 . So, there must emerge a shock as a domain boundary. The position of the shock along the backbone is denoted by $i_{S}$, which is related to the overall particle density as

$$
\rho=1-\frac{i_{S}}{L} .
$$

Making use of the shock structure, the quantity $Q_{i}^{\text {out }}$ in (29) is given by

$$
\begin{aligned}
Q_{i}^{\text {out }} & =\rho_{i-1}+\frac{2 p}{L} \sum_{j=i_{S}}^{i-2} \frac{1}{1+2 p(L-j-1) / L} \\
& =\rho_{i-1}+\ln (1+2 p \rho)
\end{aligned}
$$


with $\mathcal{O}\left(L^{-1}\right)$ corrections being ignored. Correspondingly, the particle creation probability near the exit is given by

$$
W_{c}^{\text {out }}(\rho)=\ln (1+2 p \rho) \text {. }
$$

So far we have established the shock state. Effectively, particles near the entrance hop to the right with the probability $W_{h}^{i n}$ or are annihilated with the probability $W_{a}^{i n}(\rho)$. This dynamics results in a density profile $\left\{\rho_{i}^{i n}\right\}$ which decays to zero as $i$ increases. Near the exit, particles are created effectively with the probability $W_{c}^{\text {out }}(\rho)$ and hop to the right with the probability $W_{h}^{\text {out }}$. This results in a density profile $\left\{\rho_{i}^{\text {out }}\right\}$ which converges to unity as $i$ decreases from $L$. Both profiles should be matched at a position $i_{S}=(1-\rho) L$ to yield a shock.

This situation is similar to the driven 1D ASEP with particle creation and annihilation [23, 24, 25] or boundary driven multi-lane systems (see e.g. 229, 30]). When the creation and annihilation rates are spatially uniform and inversely proportional to the lattice size, the system also develops a shock in the stead-state density profile [23, 24, 25. In comparison with the model studied in $[23,24,25,29$, , 30, the creation and annihilation rates are not uniform in space: Particles are annihilated near the entrance and created near the exit. The difference results in the feature that the shock separates the empty and the fully jammed domains.

The parameters $W_{a}^{\text {in }}(\rho)$ and $W_{c}^{\text {out }}(\rho)$ depend on the the overall particle density $\rho=1-i_{S} / L$, which should be determined self-consistently. The overall density can be obtained from the current conservation, which will be explained in the following subsection.

\subsection{Phase diagram}

Particles are injected at the entrance $(i=1)$, move to the right, and are removed at the exit $(i=L)$. So, the system can carry a nonzero current. The incoming current at the entrance and outgoing current at the exit are given by

$$
\begin{aligned}
& J_{\text {in }}(\rho)=\alpha\left(1-\rho_{1}^{\text {in }}\right) \\
& J_{\text {out }}(\rho)=\beta \rho_{L}^{\text {out }} .
\end{aligned}
$$

Because $\rho_{1}^{\text {in }}$ and $\rho_{L}^{\text {out }}$ are governed by the $\rho$-dependent effective dynamics, the incoming and the outgoing currents are given as a function of $\rho$. Particle number conservation requires that the incoming and outgoing currents should be the same in the steady state. The equality $J^{\text {in }}(\rho)=J^{\text {out }}(\rho)$ determines the overall particle density $\rho$, hence the phase diagram.

The effective dynamics is still too complex and does not allow for the closedform solution for $\rho_{i}^{\text {in,out }}(\rho)$. Therefore, the exact phase diagram will be obtained from numerical solutions of the self-consistent equations and the current-balance condition. Before doing so, we apply an approximate scheme to the self-consistent equations in order to gain a physical insight.

In terms of the effective dynamics, all particles introduced at the entrance have to be transferred toward the exit and therefore annihilated from the entry area. So, the incoming current can also be written as

$$
J_{i n}(\rho)=W_{a}^{i n} \sum_{i \geq 1} \rho_{i}^{i n} .
$$


Using the explicit forms given in (24) and (25), the self-consistent equation for the density becomes as

$$
\rho_{i}^{i n}=\frac{\rho_{i-1}^{i n}}{1+2 p(1-\rho)+\left(\rho_{i-1}^{i n}-\rho_{i+1}^{i n}\right)}
$$

for $i \geq 2$. Because of the continuous annihilation of particles, we expect that $\rho_{i}^{i n}$ decays monotonically and rather fast to zero. In order to gain a qualitative understanding we can ignore $\left(\rho_{i-1}^{i n}-\rho_{i+1}^{i n}\right)$ in the denominator to obtain that

$$
\rho_{i}^{i n} \simeq \frac{\rho_{1}^{i n}}{(1+2 p(1-\rho))^{i-1}} .
$$

Inserting these approximate solutions into (36), one obtains that

$$
J_{i n} \simeq \frac{1+2 p(1-\rho)}{1+2 p} \rho_{1}^{i n} .
$$

Comparing the two expressions for $J_{i n}$ given in (34) and (39), one finds a solution for $\rho_{1}^{i n}(\rho)$, which yields that

$$
J_{i n}(\rho) \simeq \frac{\alpha(1+2 p-2 p \rho)}{(1+\alpha)(1+2 p)-2 p \rho} .
$$

It is a decreasing function of $\rho$ with $J_{i n}(0)=\frac{\alpha}{1+\alpha}$ and $J_{i n}(1)=\frac{\alpha}{1+\alpha(1+2 p)}$.

One can carry out a similar analysis to obtain an approximate expression for $J_{\text {out }}(\rho)$. First, the outgoing current given in (35) should be equal to the total particle creation rate, that is to say,

$$
J_{\text {out }}=W_{c}^{\text {out }} \sum_{i \leq L}\left(1-\rho_{i}^{\text {out }}\right) .
$$

It is easy to show that the void density $\left(1-\rho_{i}^{\text {out }}\right)$ satisfies self-consistent equations

$$
1-\rho_{i}^{\text {out }}=\frac{1-\rho_{i+1}^{\text {out }}}{1+\ln (1+2 p \rho)+\left(\rho_{i-1}^{\text {out }}-\rho_{i+1}^{\text {out }}\right)}
$$

for $i<L$. Again the transport of particles via long ranged links can be understood as spontaneous creation of particles at sites close to the exit. Therefore, we expect that $\left(1-\rho_{i}^{\text {out }}\right)$ decays to zero as $i$ decreases from $L$. So we can ignore $\left(\rho_{i-1}^{\text {out }}-\rho_{i+1}^{\text {out }}\right)$ in the denominator as in the previous case. A similar calculation then yields

$$
J_{\text {out }}(\rho) \simeq \frac{\beta(1+\ln (1+2 p \rho))}{1+\beta+\ln (1+2 p \rho)} .
$$

This is an increasing function of $\rho$ with $J_{\text {out }}(0)=\frac{\beta}{1+\beta}$ and $J_{\text {out }}(1)=\frac{\beta(1+\ln (1+2 p))}{1+\beta+\ln (1+2 p)}$.

The particle number conservation requires that the incoming current and the outgoing current should be the same in the steady state. Figure 6 shows schematic plots of $J^{\text {in }}(\rho)$ and $J^{\text {out }}(\rho)$ in three different situations. In the first case, the current curves may intersect with each other at $\rho=\rho_{0}$ with $0<\rho_{0}<1$ as shown in Figure 6 (a). This case corresponds to the shock phase. The intersection determines the shock position $i_{S}=\left(1-\rho_{0}\right) L$. If $\rho$ becomes greater (smaller) than $\rho_{0}$ due a stochastic fluctuation, then the incoming current becomes smaller (greater) than the outgoing current. Consequently, the particle density is attracted toward the steady-state value and the shock is driven toward the steady-state position. This explains the reason why there is a localized sharp shock [23, 24, 25]. 

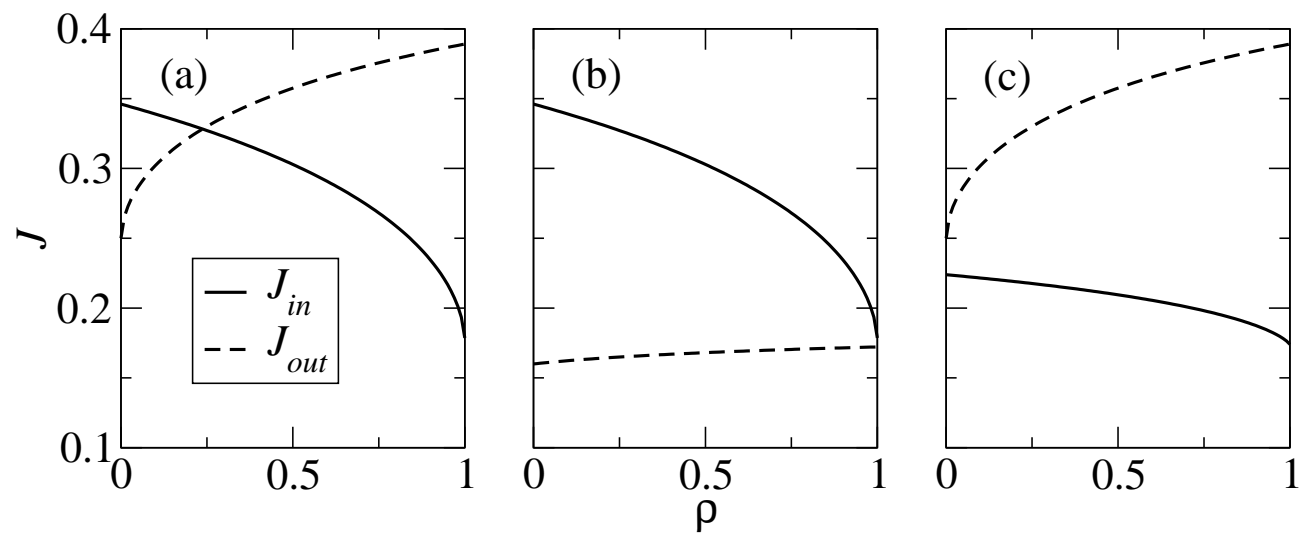

Figure 6. Schematic plots of $J_{\text {in }}$ (solid lines) and $J_{\text {out }}$ (dashed lines) against $\rho$.

The second case with $J_{\text {in }}(\rho)>J_{\text {out }}(\rho)$ for all values of $\rho$ is shown in Figure 6(b). Then, the shock is localized at the entrance and the steady-state density is equal to one. In this case the system belongs to the jammed phase.

The third case is sketched in Figure 6(c). If $J_{\text {in }}(\rho)<J_{\text {out }}(\rho)$ for all values of $\rho$, the shock is localized at the exit and the steady-state density is equal to zero. Then the system belongs to the empty phase.

We have solved numerically exactly the self-consistent equations for $\rho_{i}^{\text {in }}$ and $\rho_{i}^{\text {out }}$ to obtain the incoming and outgoing currents as a function of $\rho$ at each set of values of $\alpha, \beta$, and $p$. The current balance condition allows us to evaluate the steady-state particle density and the current, hence the phase diagram. The resulting numerical phase diagram in the $\alpha \beta$ plane is presented in Figure 4 . The phase diagram at $p=0.2$ consists of the empty phase, jammed phase, and the shock phase, which is consistent with the Monte Carlo result. There is a quantitative discrepancy in the location of the phase boundaries, which is caused by the approximations.

The annealed approximation requires the self-averaging property [31, 32. The self-averaging property in the small-world network was tested for the equilibrium Ising model [32. To test the self-averaging property in our nonequilibrium model, we have measured the relative sample-to-sample fluctuation of the particle density $X \equiv \sqrt{\left(\left[\left\langle\frac{1}{L} \sum_{i} n_{i}\right\rangle^{2}\right]_{\mathcal{G}}-\left[\left\langle\frac{1}{L} \sum_{i} n_{i}\right\rangle\right]_{\mathcal{G}}^{2}\right)} /\left[\left\langle\frac{1}{L} \sum_{i} n_{i}\right\rangle\right]_{\mathcal{G}}$. In the $\mathrm{E}$ and J phases, it decays to zero as $X \sim L^{-1 / 2}$ suggesting a strong self-averaging [31]. On the other hand, it converges to a finite value in the $\mathrm{S}$ phase, an indication of non-self-averaging. This tells us that the annealed approximation has a limitation. It explains successfully the mechanism of shock formation, but not its average position due to the strong fluctuations. A refined approach beyond the annealed approximation is necessary to study the sample-to-sample fluctuation phenomena in the S phase.

\section{Summary and Conclusion}

We have studied the boundary-driven ASEP in the 1D chain with long-range links. This setup represents a generic situation for directed transport in a complex network, e.g., the exchange of data between two sites of a computer network.

The backbone of nearest neighbor links ensures the existence of a path between 
start and destination. The long ranged links add shortcuts to the transport network that are in principle able to enhance the capacity of the system.

Considering the typical results for transport problems on complex networks one would also expect that long-range shortcuts, added randomly on to a lattice, are believed to suppress fluctuations and make a system homogeneous. This is, however, not the case when there is a boundary driving. Compared to the pure one-dimensional system it turns out that the long-range links play an essential role. They generate a localized shock which separates the 1D chain backbone into an empty and jammed regions.

Adopting the mean field and the annealed approximations, we have derived effective dynamics near the entrance and the exit, which are similar to those of the ASEP with spontaneous particle annihilation and creation, respectively. The effective theory reveals the mechanism for the shock formation and for the phase transition. The phase diagram consists of the empty, shock, and jammed phases. The shock phase is characterized by presence of a localized shock and separating a low and a high density domain. The shock position and the overall particle density $\rho$ vary continuously with the model-parameters. In the empty (jammed) phase, the shock is anchored to the exit (entrance) to yield $\rho=0$ (1).

In conclusion, our study shows that a driven system on a spatially disordered structure displays an inhomogeneous pattern. The appearance of a localized shock is reminiscent of one-dimensional systems without particle-conservation in the bulk. In contrast to these systems the mechanism driving the localization of the shock is neither the competition between bulk and boundary reservoirs nor an optimal partitioning between multiple lanes. Here, the long-ranged links enhance the mass transfer between entry and exit and thereby stabilize the position of the shock.

Considering more generally the transport capacity of a complex network between arbitrary sites our results have important consequences. They indicate that, as far as the capacity of the feeding particle reservoir does not exceed the capacity of the exit reservoir, the sites of the backbone are only rarely occupied and can be used in parallel for transport issues between other sites. Contrary, overfeeding the backbone leads to a complete blockage of the sites in question which may spread over the whole network.

\section{Acknowledgments}

This work was supported by Mid-career Researcher Program through NRF grant (No. 2010-0013903) funded by the MEST.

\section{References}

[1] Derrida B 1998 Phys. Rep. 30165

[2] MacDonald C T, Gibbs J H and Pipkin A C 1968 Biopolymers 6 1; MacDonald C T and Gibbs J H 1969 Biopolymers 7707

[3] Spohn H 2006 Physica A 36971

[4] Nagel K and Schreckenberg M 1992 J. Phys. I 22221

[5] Chowdhury D, Santen L and Shadschneider A 2000 Phys. Rep. 329199

[6] Gwa L -H, Spohn H 1992 Phys. Rev. A 46844

[7] Derrida B, Evans M R, Hakim V and Pasquier V 1993 J. Phys. A: Math. Gen. 261493

[8] Schütz G M and Domany E 1993 J. Stat. Phys. 72277

[9] Derrida B and Lebowitz J L 1998 Phys. Rev. Lett. 80209

[10] Lee D -S and Kim D 1999 Phys. Rev. E 596476 
[11] de Gennes P G 1979 Scaling Concepts in Polymer Physics (Ithaca: Cornell University Press)

[12] Berg O G, Winter R B and von Hippel P H 1981 Biochemistry 206929

[13] Watts D J and Strogatz S H 1998 Nature 393440

[14] Albert R and Barabási A -L 2002 Rev. Mod. Phys. 7447

[15] Szavits-Nossan J and Uzelac K 2006 Phys. Rev. E 74 051104; Szavits-Nossan J and Uzelac K 2008 Phys. Rev. E 77051116

[16] Ha M, Park H, and den Nijs M 2997 Phys. Rev. E 75061131

[17] Otwinowski J and Boettcher S 2009 J. Stat. Mech. P07010

[18] Edwards S F and Wilkinson D R 1982 Proc. R. Soc. A 38117

[19] Kardar M, Parisi G and Zhang Y C 1986 Phys. Rev. Lett. 56889

[20] Kim D 1995 Phys. Rev. E 523512

[21] Dorogovtsev S N, Goltsev A V, and Mendes J F F 2009 Rev. Mod. Phys. 801275

[22] Privman V 1990 Finite Size Scaling and Numerical Simulation of Statistical Systems (Singapore: World Scientific)

[23] Parmeggiani A, Franosch T, and Frey E 2003 Phys. Rev. Lett. 90086601

[24] Evans M R, Juhász R, Santen L 2003 Phys. Rev. E 68026117

[25] Juhász R and Santen L 2004 J. Phys. A: Math. Gen. 373933

[26] Boguñá M, Castellano C and Pastor-Satorras R 2009 Phys. Rev. E 79036110

[27] Noh J D and Park H 2009 Phys. Rev. E 79056115

[28] Lee S H, Ha M, Jeong H, Noh J D and Park H 2009 Phys. Rev. E 80051127

[29] Reichenbach T, Frey E and Franosch T 2006 Phys. Rev. Lett. 97050603

[30] Schiffmann C, Appert-Rolland C and Santen L 2010 J. Stat. Mech. P06002

[31] Wiseman S and Domany E 1998 Phys. Rev. Lett. 8123

[32] Roy S and Bhattacharjee S M 2006 Phys Lett A 35213 